\title{
The Value of T-SPOT.TB Technology in the Diagnosis of Tuberculosis in Elderly Patients

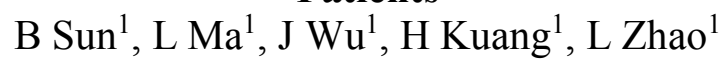

\begin{abstract}
Objectives: To investigate the clinical value of T-SPOT.TB technology $(\gamma$-interferon release assay for tuberculosis (TB) infection in the diagnosis of TB in elderly patients.

Methods: One-hundred forty out patients and in patients diagnosed with TB in our hospital from February 2013 to February 2014 were classified into a young adult group or an elderly group. Patients in both groups underwent peripheral blood T-SPOT.TB tests and the purified protein derivative test (tuberculin test), and the results were analyzed.

Results: The T-SPOT. TB test positive rate in the young patient group was $90.3 \%$, significantly higher than the PPD test positive rate of $63.9 \%$. Similarly, the T-SPOT.TB positive rate in the elderly group was also significantly higher than the PPD test positive rate (88.2\%vs. 39.7\%).When these two detection methods were compared, T-SPOT.TB had a significantly higher detection rate than the PPD test in both the young adult group and the elderly group. The difference was statistically significant in both groups and was especially pronounced in the elderly group.
\end{abstract}

Conclusions: As a new diagnostic technique, the T-SPOT.TB demonstrated good value in the clinical diagnosis of tuberculosis in the elderly group.

Keywords: Elderly, interferon release assay, tuberculosis, $\gamma$ - tuberculin test

From: Department of Respiratory Medicine, Henan Provincial people's hospital (the People's Hospital of Zhengzhou University), Zhengzhou, China. 450000

Correspondence: Dr L Zhao, Henan Provincial People's Hospital, The People's Hospital of Zhengzhou University, No.7, Wei Wu Road, Zhengzhou, Henan, China. Fax: +86-037165964376; e-mail: liminzhao889@sina.com 


\section{INTRODUCTION}

Tuberculosis (TB) poses a serious threat to human health. It is ranked as the top cause of death due to infectious diseases. Although once effectively controlled, its incidence worldwide in the past 20 years has risen rapidly, especially in developing countries. The prevalence of disease is positively correlated to age. Elderly patients with tuberculosis are especially at risk due to weakness, decreased immune function, and comorbidities such as diabetes, cardiovascular disease, and cancer. These factors can cause latent TB to resurface, and the possibility of re-infection of TB in this group is significantly higher than in younger people. These factors also contribute to atypical clinical manifestations and imaging changes.

The sensitivity and specificity of purified protein derivative (PPD) and other supplemental screening methods are poor for elderly patients, increasing the difficulty of TB diagnosis. Finding rapid and sensitive diagnostic indicators for elderly patients with TB is of great significance. In recent years, the clinical use of T-SPOT.TB in tuberculosis detection, including the diagnosis of TB in the incubation period, was widely acknowledged (1-2). However, few studies of its role in the diagnosis of pulmonary TB in elderly patients have been done. In this study, we evaluated T-SPOT.TB's application and clinical value in the diagnosis of pulmonary TB in elderly patients.

\section{MATERIALS AND METHODS}

Clinical data: One-hundred forty patients with active TB diagnosed at Henan Province People's Hospital inpatient and outpatient departments from February 2013 to February 2014 were selected. Among them, there were 88 men and 52 womenaged22 to 86 years (average age, 53). These subjects were classified into two age-groups: the elderly group, with patients older than 60 years, and the young adult, with patients aged 18 to 60 years. 
Inclusion criteria: Diagnosis of TB was based on the China TB Control \& Implementation Program Guide (2008 Edition) (3). Exclusion criteria: (1) Anti-TB drugs used in the past 3 days; (2) Unstable vital signs, including circulatory failure and respiratory failure or a required intensive care unit stay due to critical condition; and (3) Lack of cooperation and poor compliance. The following information was documented for each subject: (1)History of TB and anti-TB treatment; (2) Basic health history, including respiratory system illness such as chronic obstructive pulmonary disease and bronchial asthma and chronic non-respiratory diseases such as diabetes, coronary heart disease, chronic renal insufficiency, connective tissue disease, blood disease, and AIDS; (3) Whether corticosteroid and/or an immunosuppressant was used 3 months before enrollment; (4) Important clinical findings at enrollment (white blood cell count, lymphocyte count, serum total protein, serum albumin);(5) Imaging findings (eg, exudation, consolidation, cavities); and (6) Whether the subject received Bacillus Calmette-Guérin(BCG) vaccination. All subjects with active TB underwent PPD and T-SPOT.TB tests after diagnosis and before treatment; blood samples were collected for the T-SPOT.TB testing before the PPD test.

\section{METHODS}

T-SPOT.TB test: The diagnosis kit was provided by Shanghai Fuxing Science and Technology Co., Ltd. Five milliliters of venous blood were collected with heparin; specific steps can be found in the product manual. Result interpretation: When A or B test wells met the following criteria, it was judged a positive result: (1) When the number of spots in the negative control wells was between 0 and 5, the number of spots in the test wells minus the number of spots in negative control wells was greater than or equal to 6; and (2) When the 
number of spots in negative control wells was greater or equal to 6 , the number of spots in the test wells should have been at least double the spots in the negative control wells.

Tuberculin skin (PPD) test: The PPD was diluted with sterile saline into different concentrations. $0.1 \mathrm{ml}(5 \mathrm{IU})$ was injected into the front left volar forearm skin one-third of the way to the center. The reaction was checked 72 hours later (induration was noted; skin flush cannot be used as the sole standard). The evaluation criteria refers to the China TB Control \& Implementation Program Guide (2008 Edition) standard (3). Induration diameter (d) $<5 \mathrm{~mm}$ is regarded as negative (-); 5 to $10 \mathrm{~mm}$ is weakly positive $(+)$; and 10 to 20 is positive $(++)$. If the diameter is $\geq 20 \mathrm{~mm}$ or there are local blisters, necrosis, or lymph node inflammation, they are all strongly positive $(+++)$.

Statistical analysis: All data were analyzed by SPSS17.0 statistical software. Measurement data were analyzed using t-test statistical analysis, while count data were analyzed using the chi-square test; $P \geq 0.05$ was considered statistically significant.

\section{RESULTS}

Clinical data: There were 68 patients with active TB in the elderly group, including 40 men and 28 women. The average age was 72 years. Forty-six patients had secondary pulmonary TB, 2 had miliary TB, 6 had TB pleurisy, 8 had pulmonary TB and TB pleurisy, and 6 had lymph node TB. TB was diagnosed in 9 patients in this group by acid-fast staining of sputum smears. Eight patients were diagnosed by acid-fast staining of bronchoalveolar lavage fluid, and 34 patients were diagnosed through an acid-fast staining test of transbronchial and lung biopsy and/or TB-DNA. Pleural biopsyacid-fast staining and/or TB-DNA confirmed TB in 11 patients. Lymph node biopsy acid-fast staining and/or TB-DNA detection confirmed TB in 6 patients. 
There were 72 patients with active TB in the young adult group, including 48 men and 24 women who were, on average, 38 years old. Among them, 38 patients had secondary pulmonary TB, 1 patient had miliary TB, 11 patients had tuberculous pleurisy, 16 patients had pulmonary TB and tuberculous pleurisy, and 6 patients had lymph node TB. TB was diagnosed in 16 patients in this group by acid-fast staining of sputum smears, 14 patients were diagnosed by acid-fast staining of bronchoalveolar lavage fluid, and 19 patients were diagnosed through acid-fast staining test of transbronchial and lung biopsy and/or TB-DNA.

Pleural biopsy acid-fast staining and/or TB-DNA aided diagnosis in 17 patients. Lymph node biopsy acid-fast staining and/or TB-DNA detection confirmed the diagnosis in 6 patients. (See Table 1 for specific subject-relevant clinical data.)Statistical analysis showed that there were significant differences between these 2 groups in both existing respiratoryrelated diseases and non-respiratory diseases. The prevalence of existing diseases in the elderly group was significantly higher than in the young adult group. In addition, BCG vaccination rates in the elderly group were significantly lower than in the young adult group.

Both groups of patients underwent PPD and T-SPOT.TB tests. In the young adult group, the T-SPOT.TB test positive rate was $90.3 \%$, significantly higher than the PPD test positive rate of $63.9 \%$. Similarly, for the elderly patients, the T- SPOT.TB test positive rate $(88.2 \%)$ was also significantly higher than the PPD positive rate $(39.7 \%)$. When these two detection methods were compared, T-SPOT.TB positive rates were statistically significantly higher than PPD test positive rates in both the young adult and the elderly groups. The difference was more pronounced in the elderly group (Table 2).

The PPD positive rate in the elderly group was $39.7 \%$, significantly lower than the PPD-positive rate in the young adult group (63.9\%); the difference was statistically significant. The T-SPOT.TB positive rate in the young adult group $(90.3 \%)$ was higher than in the elderly group $(88.2 \%)$, but this difference was not statistically significant. Negative results were 
observed with both detection methods. There was no significant difference in the T-SPOT.TB test negative rate in the elderly group (7.4\%) and the young adult group (6.9\%). However, the PPD negative rate in the elderly group $(60.3 \%)$ was significantly higher than in the young adult group (36.1\%). Two patients could not be diagnosed by the T-SPOT.TB test in the elderly group (2.9\%).Three patients in the young adult group could not be determined(4.2\%). There was no significant difference (Table 3).

\section{DISCUSSION}

With the acceleration of aging in our country, there have been widespread and more severe cases of TB in the elderly population. The fifth national TB epidemiological survey data showed that TB prevalence among persons 60 years and older has skyrocketed to 2 to 3 times that of the general population (4). While TB clinical symptoms are not typical in elderly persons, the smear-positive rate was as high as $36.75 \%(5)$, and the smear-positive rate among persons 60 to 75 years old has continued to rise in recent years. In addition, $\mathrm{x}$-ray findings are not typical for elderly TB patients, who also have more comorbidities. Thus, TB not only seriously endangers the health of elderly persons, but it is likely to become an important source of infection in families and communities. With the progress of social civilization, the world's aging population has become more evident. Paying attention to elderly TB patients has important practical significance.

There are many ways to diagnose TB, among which the tuberculin test is still one of the most important ways. However, because PPD is a mixture of various protein components, it is susceptible to the influence of BCG vaccination and environmental mycobacterial infections, and its specificity to TB and the detection rate of M. TB infections are low. The TSPOT.TB test uses M. TB-specific antigen technology and thus was not susceptible to the 
impact of BCG vaccination and other non-tuberculous mycobacterial infections. The specificity was significantly improved compared with that of PPD. A meta-analysis of existing reports showed that PPD sensitivity in TB patients was $67 \%$ to $72 \%$ (2), and the sensitivity of T-SPOT.TB in TB patients was $85 \%$ to $90 \%$ (6).

Other scholars have reported that PPD specificity in TB patients was $52.2 \%$ to $84.4 \%$ and the specificity of T-SPOT.TB was as high as94.1\% (7). In addition, an immunocompromised host or immune suppression has little impact on T-SPOT.TB testing; it maintains high sensitivity and specificity in immunosuppressed individuals such as AIDS patients, patients with connective tissue disease, and infants and young children (8). This study also found that the T-SPOT.TB had a high positive rate in both the elderly group (88.2\%) and the young adult groups $(90.3 \%)$.

PPD positive rates were lower than in the T-SPOT.TB in both the elderly and the young adult groups; the difference was statistically significant, which is similar to what has been reported in other studies. Furthermore, we found that the PPD positive rate in the older group was significantly lower than in the young adult group, which is consistent with domestic research results $(9-12)$. The reason may be that immune function significantly decreases in elderly persons, and cellular immune function, especially $\mathrm{T}$ lymphocyte function, is closely related to PPD test results. Thus, in elderly patients with $\mathrm{TB}$, reaction to the PPD test weakens. However, the T-SPOT.TB positive rate significantly increased in elderly patients, and these positive rates were not significantly different from what we saw in the young adult group, suggesting that T-SPOT.TB testing effectiveness was not negatively affected by older age and decreased immune function.

In this study, $6 \mathrm{~TB}$ patients in the elderly group were diagnosed as negative by the TSPOT.TB test, and 2 patients were not diagnosed. Similar results were also observed in the young adult group. This corresponded well to the latest systematic review of $3.8 \%$ (95\% CL, 
$3.5 \%-4.3 \%$ ) uncertainty(13). The reason for the uncertainty is mainly a higher negative background, or the number of spots against overlapping peptide antigen is in the cut-off range, which makes it difficult to diagnose in the clinical setting. Further analysis of elderly patients with negative or undetermined T-SPOT.TB results found that, in the 2 undetermined patients, peripheral lymphocyte counts and ratios were lower than normal, which may be related to chronic underlying diseases, old age, nutritional status, and other factors (Table 4).Because the T-SPOT.TB test targets antigen-specific memory $\mathrm{T}$ cells infected with $\mathrm{TB}$, reduction of the total number of lymphocytes may lead to ambiguous interpretation of the results. In the 6 patients with negative results, the peripheral blood lymphocyte count also declined but only slightly. In addition, sputum-smear tests of these 6 patients were negative, and 5 of these patients had diabetes. Whether these factors are related to the T-SPOT.TB negative results still needs more in-depth study in a larger sample size. Although relevant research suggests that diabetes does not affect the sensitivity of T-SPOT.TB in the diagnosis of TB (14-16), none of these studies of TB infections in diabetic patients included well-designed groups, and the sample size was small. Thus, the significance of T-SPOT.TB in the diagnosis of TB in diabetic patients still needs more in-depth discussion.

In summary, elderly TB patients are a special population because of their impaired humoral and cellular immune functions and comorbidities such as diabetes and COPD, which result in a lower TB detection rate. Traditional test methods such as PPD and TB antibody have poor specificity and sensitivity and cannot meet clinical diagnosis needs. As a rapid, non-invasive, simple method to diagnose TB, T-SPOT.TB has not been evaluated for its diagnostic value in elderly patients. This study found that T-SPOT.TB had the highest specificity in elderly TB patients. It is an important method for diagnosis of TB with high diagnostic value and is worthy of further promotion in the clinic setting. 


\section{ACKNOWLEDGMENTS}

This study don't have any fund supported.

\section{AUTHORS' NOTE}

There are no conflict of interests associated with this study. 


\section{REFERENCES}

1. Lui G, Lee N, Cheung SW, Lam JS, Wong BC, Choi KW, Wong KT, Wong RY, Cockram CS, Hui DS, Chan RC. Interferon gamma release assay for differentiating tuberculosis among pneumonia cases in acute healthcare setting. J Infect 2011; 62: $440-447$.

2. Diel R, Loddenkemper R, Nienhaus A. Evidence-based comparison of commercial interferon-gamma release assays for detecting active TB: a meta analysis.Chest 2010; 137: 952-968.

3. Ministry of Health, Bureau of Disease Control and Medical Administration, Chinese Center for Disease Control and Prevention. China TB Control and Implementation Programs Guide (2008 edition) Beijing: China Union Medical University Press, 2009.

4. The Fifth National TB Epidemiological Survey in 2010. Chin J Tuberculosis Prevention 2012; 8: 485-508.

5. Chen H. Analyses of Clinical Data and Missed Diagnosis in elderly patients with TBpositive Tuberculosis. Chin J Infection Control 2006; 5: 327-329.

6. Sadatsafavi M, Shahidi N, Marra F, FitzGerald MJ, Elwood KR, Guo N, Marra CA. A statistical method was used for the meta-analysis of tests for latent TB in the absence of a gold standard, combining random-effect and latent-class methods to estimate test accuracy. J Clin Epidemiol 2010; 63: 257-269.

7. Zhang, LH, Jin WX, Jian HY. Clinical Diagnostic Value of T-SPOT and Tuberculin Test for tuberculosis patients. Chinese J. Clinicians 2012; 7: 4107-4108.

8. Cruz AT, Geltemeyer AM, Starke JR, Flores JA, Graviss EA, Smith KC. Comparing the tuberculin skin test and T-SPOT.TB blood test in children. Pediatrics 2011; 127 : e31-38.

9. Liu JG, Qiao YH, Liu XQ. The PPD Test Reaction Intensity Analyses in 30 Cases 
of Elderly Tuberculosis Patients, J of Practical Diagnosis and Therapy 2003; 17:381382.

10. James H. Cutaneous testing in the elderly patient with tuberculosis. Chest $1980 ; 77$ : 188-189.

11. Yang Y. Elderly tuberculosis. Chinese Journal of Tuberculosis and Respiratory Diseases1996; 19:199.

12. Ozekinci T, Ozbek E, Celik Y. Comparison of tuberculin skin test and a specific Tcell-based test, T-Spot. TB, for the diagnosis of latent tuberculosis infection $\mathrm{J}$ Int. Med Res 2007; 35: 696-703.

13. Pai M, Riley LW, Colford JM. Interferon-gamma assays in the immunodiagnosis of tuberculosis: a systematic review. Lancet Infect Dis 2004; 4:761-776.

14. Gao MQ, Liu F, Ji BY. Comparative Analysis of two $\gamma$ - Interferon Release Assays in the Clinical Diagnosis of Tuberculosis. Chinese J Tuberculosis and Respiratory Diseases 2012; 35:762-765.

15. Liu RM, Gao MQ, Zhang LQ. The diagnostic value of $\gamma$ - Interferon Release Assayin diabetic patients with tuberculosis. Chinese Journal of Medicine 2014; 49:8687.

16. Tan CK, Lai CC, Chen HW, Liao CH, Chou CH, Huang YT, Yang WS, Yu CJ, Hsueh PR. Enzyme-linked immunospot assay for interferon-gamma to support the diagnosis of tuberculosis in diabetic patients. Scand J Infect Dis. 2010; 42: 752-756. 
Table 1: Clinical data of study subjects

\begin{tabular}{|c|c|c|c|c|c|c|c|c|}
\hline \multirow{3}{*}{\multicolumn{2}{|c|}{ Clinical Characteristics }} & Elderly Grc & & & Young Ad & It Group & & \multirow{4}{*}{$\begin{array}{l}P \text { value } \\
<0.001\end{array}$} \\
\hline & & $\begin{array}{l}\text { Tubercul } \\
\text { osis }\end{array}$ & $\begin{array}{l}\text { Extrapulmo } \\
\text { nary } \\
\text { tuberculosis }\end{array}$ & Total & $\begin{array}{l}\text { Tubercul } \\
\text { osis }\end{array}$ & $\begin{array}{l}\text { Extrapul } \\
\text { monary } \\
\text { tubercul } \\
\text { osis }\end{array}$ & Total & \\
\hline & & $n=48$ & $n=20$ & $n=68$ & $n=39$ & $n=33$ & $\mathrm{n}=72$ & \\
\hline \multicolumn{2}{|l|}{ Age } & $\begin{array}{l}72.73 \pm 5 \\
54\end{array}$ & $73.20 \pm 6.06$ & $\begin{array}{l}72.87 \pm 5 \\
65\end{array}$ & $\begin{array}{l}35.05 \pm 9 . \\
08\end{array}$ & $\begin{array}{l}33.03 \pm 8 \\
69\end{array}$ & $\begin{array}{l}34.13 \pm 8 \\
90\end{array}$ & \\
\hline \multirow{2}{*}{ Gender } & Male & 26 & 14 & 40 & 25 & 23 & 48 & \multirow{2}{*}{0.337} \\
\hline & Female & 22 & 6 & 28 & 14 & 10 & 24 & \\
\hline \multicolumn{2}{|c|}{ History of Smoking (\%) } & $28(58.3)$ & $13(65.0)$ & $41(60.3)$ & $22(56.4)$ & $20(60.6)$ & $42(58.3)$ & 0.813 \\
\hline Existi & Respiratory（\%） & $10(20.8)$ & $7(35.0)$ & $17(25.0)$ & $3(7.7)$ & $2(6.1)$ & $5(6.9)$ & 0.003 \\
\hline $\begin{array}{l}\text { ng } \\
\text { disea } \\
\text { ses }\end{array}$ & $\begin{array}{l}\text { Non-respiratory } \\
(\%)\end{array}$ & $21(43.8)$ & $11(55.0)$ & $32(47.1)$ & $4(10.3)$ & $6(18.2)$ & 10 (13.9) & $<0.001$ \\
\hline \multicolumn{2}{|c|}{ Use of Glucocorticoids (\%) } & $4(8.3)$ & $2(10)$ & $6(8.8)$ & 7 (17.9) & $7(21.1)$ & $14(19.4)$ & 0.073 \\
\hline \multicolumn{2}{|c|}{$\begin{array}{l}\text { Bacillus Calmette- } \\
\text { Guérinvaccination (\%) }\end{array}$} & $6(12.5)$ & $2(10)$ & $8(11.8)$ & $32(82.1)$ & 29 (87.9) & $61(84.4)$ & $<0.001$ \\
\hline \multicolumn{2}{|c|}{ Anti-TB Treatment (\%) } & $12(42.9)$ & $9(45)$ & $21(30.9)$ & $8(20.5)$ & $9(27.3)$ & $17(23.6)$ & 0.334 \\
\hline
\end{tabular}


Table 2: Detection results comparison using purified protein derivative test and T-SPOT.TB in both groups

PPD Test

$\begin{array}{lll}\text { Positive } & \text { Negative } & \text { Total }\end{array}$

\begin{tabular}{|c|c|c|c|c|c|c|}
\hline \multirow{5}{*}{$\begin{array}{l}\text { T-SPOT.TB } \\
\text { Test }\end{array}$} & \multirow{5}{*}{$\begin{array}{l}\text { Elderly } \\
\text { Group }\end{array}$} & \multirow{4}{*}{$\begin{array}{l}\text { Young } \\
\text { Adult }\end{array}$} & \multicolumn{2}{|r|}{ Young } & \multirow[b]{2}{*}{ Elderly } & \multirow[t]{2}{*}{ Young } \\
\hline & & & Elderly & & & \\
\hline & & & & Adult & & Adult \\
\hline & & & Group & & Group & \\
\hline & & Group & & Group & & Group \\
\hline Positive & 39.7 & 63.9 & 48.5 & 26.4 & 88.2 & 90.3 \\
\hline Negative & 0 & 0 & 7.4 & 6.9 & 7.4 & 6.9 \\
\hline Undetermined & 0 & 0 & 2.9 & 4.2 & 2.9 & 4.2 \\
\hline Total & 39.7 & 63.9 & 60.3 & 36.1 & 100 & 100 \\
\hline
\end{tabular}

PPD, purified protein derivative. 
Table 3. Detection results comparison using purified protein derivative and T-SPOT.TB in both groups

\begin{tabular}{cccccc}
\hline & & Elderly Group & Young Group & Total & $P$ value \\
& & $(\mathrm{n}=68)$ & $(\mathrm{n}=72)$ & $(\mathrm{n}=140)$ & \\
\hline \multirow{2}{*}{ PPD Test } & Positive & 27 & & & \\
& Negative & 41 & 26 & 67 & 0.003 \\
T- & Positive & 60 & 65 & 125 & 0.710 \\
SPOT.TB & Negative & 6 & 4 & 10 & 0.708 \\
Test & Undeter & & & & \\
& mined & 2 & 3 & 5 & 0.913 \\
& & & & & \\
\hline
\end{tabular}


Table 4: Undetermined or negative cases using the T-SPOT.TB test in the elderly group.

T-

Num

ber

SPOT.TB Age Comorbidity

Results
White cell Lymphocyte PP

count s Count D

Diagnosis

Type II

Diabetes

$1 \quad$ Negative $\quad 76$

$4.6 \quad 1.0 \times 10^{9} / \mathrm{L} \quad-\quad$ Tuberculosis

Coronary

Heart Disease

2 Negative $68 \quad$ COPD

$5.2 \quad 0.8 \times 10^{9} / \mathrm{L}$

Tuberculous

pleurisy

Type II

$3 \quad$ Negative 67

$2.9 \quad 0.8 \times 10^{9} / \mathrm{L}$

Tuberculosis

Diabetes

Type II

$4 \quad$ Negative 72

Diabetes

6.2

$1.1 \times 10^{9} / \mathrm{L}$

Tuberculosis

Rheumatoid

Arthritis

$5 \quad$ Negative 66

3.8

$0.7 \times 10^{9} / \mathrm{L}$

Tuberculosis

Type II

Diabetes

Lung cancer

$6 \quad$ Negative $75 \quad$ Type II

$5.4 \quad 0.9 \times 10^{9} / \mathrm{L} \quad-$

Tuberculous

Diabetes 
Not

7 Determine 73 Hypertension $7.3 \quad 0.5 \times 10^{9} / \mathrm{L} \quad$ - $\quad$ Tuberculosis

d

$8 \quad$ Not

Nephrotic

$\begin{array}{lllll}\text { Determine } & 75 & 6.4 & 0.6 \times 10^{9} / \mathrm{L} \quad & -\end{array}$ Tuberculosis

d

PPD, purified protein derivative. 